\title{
Increased Efficiency of Hydraulic Systems Through Reliability Theory and Monitoring of System Operating Parameters
}

\author{
Mitar Jocanović* - Dragoljub Šević - Velibor Karanović - Ivan Beker - Slobodan Dudić \\ Faculty of Technical Sciences, University of Novi Sad, Serbia
}

In the process of hydraulics systems design various software simulation systems are used. However, the increase of efficiency of the designed hydraulic systems can be achieved in two ways: by making design modifications based on reliability theory, on one hane, or based on monitoring of system operating parameters, on the other. In this paper, a case study of the improvement of a designed and implemented hydraulic system is reviewed, using those two approaches. Based on the data collected by monitoring the system operating parameters, and the system reliability analysis, it was possible to increase the efficiency of the hydraulic system either it the initial design stage, or during system realization. The result of such an approach is the hydraulic system which successfully operates 24 hours a day, without failure, which indicates that the proposed method of system analysis and improvement allows significant enhancement of hydraulic system efficiency.

Keywords: hydraulics, hydraulic system reliability, hydraulic system monitoring and efficiency

\section{O INTRODUCTION}

Design based on reliability is often very complex since, besides requiring statistical data on the component/system, it also depends on other, often insufficiently reliable factors which influence the considered hydraulic component or system. However, despite present uncertainty, throughout the hydraulic systems design process the analysis of statistical data can represent a very useful method for the increase of system availability and reliability, and, by extension, the effectiveness of hydraulic systems.

Analysis of hydraulic systems operation indicates that reliability of systems and components depends on a large number of various factors, which are often interrelated in a complex manner. These factors are: environmental influence, mechanical properties of used materials, wear, dynamic loads, material fatigue, time spent in regular exploitation, as well as the recommendations on maintenance and servicing of system/component. In their research, Fitch and Hong [1] show the influence of various factors on the reliability of components in the hydraulic system and they explicitly show this in their work. Savić et al. [2] are engaged in experimental research on the impact of variability of operating parameters (pressure, temperature, fluid viscosity) to the pressure drop in pipe systems of the hydraulic systems.

Operating conditions have a significant role in the reliability of a hydraulic component or system, which has been discussed in various sources dealing with statistics and reliability [3] and [4]. They directly lower the designed reliability. In his book, Savić et al. [3] defines certain operating parameters that affect e safe operation of hydraulic systems and components. Jocanović [4] studies the impact of changes of working parameters in hydraulic system (change of the values of pressure, temperature and viscosity of hydraulic fluid to the number and size of solid particles that pass through the gap in the hydraulic component) and their impact on the reliability of components in the system. Herakovic [5] theoretically analyses the occurrence of opposed forces depending of specific flow rates of hydraulic valves and experimentally shows the influence of operating parameters (pressure and flow changes) to the reliable operation of hydraulic valves. Birolini [6] and Ivanović et al. [7] determine the basic formula for calculating the reliability of technical systems used in writing this paper. Moreover, reliability of a system can be increased exclusively during the designing stage, while at every other lifecycle stage the reliability tends only towards diminishment. If a particular component is replaced by a more reliable one, then one speaks about designing an improved system, i.e., such a system is considered a completely different system from the original one. Books and papers listed above are used in the theoretical analysis of reliability and experimental resolution of issues related to the influence of working parameters on the operation of hydraulic components and systems in general, and in the process of monitoring operation of hydraulic systems.

\section{INITIAL DATA FOR RELIABILITY ANALYSIS OF A HYDRAULIC SYSTEM}

In order to review the process of hydraulic system design, and show a proper way of selecting hydraulic components, an existing machine with lower reliability record has been chosen for this experiment. Detection of critical system components has been conducted 
by monitoring the operating parameters (pressure, temperature - kept within optimal range of 40 to 60 ${ }^{\circ} \mathrm{C}$, and flow) which are presented later in the paper. The analysis of operation of hydraulic system has shown that, reliability-wise, all the components have been serially connected (in case of a failure of any component, the whole system stops). The hydraulic system is shown in Fig. 1. It is composed of three sub-systems: accumulation, extrusion, and filling subsystem, which operate in alternation.

Based on the data given by Fitch [1], who has defined the expected (most probable) failure rates for each hydraulic system component; it is possible to define the expected reliability of each of the subsystems within a considered hydraulic system. The values of failure rate for the components of the example system are presented in Table 1, as given by [1].

\section{RELIABILITY ANALYSIS OF SYSTEM IN OPERATION}

Analysis of reliability has been conducted under the assumption that the system is operating in the period of random failures (system reliability can be described with exponential distribution law) which holds that [6] and [7]:

$$
R=e^{-\lambda \cdot t},
$$

where $R$ is system reliability, $\lambda$ system failure rate (for the exponential distribution function, failure rate is constant or nearly constant value), $t$ system operational time (in Nomenclature Section symbols used in this paper are presented).

Based on the data from the literature [1], it is possible to calculate expected failure rate, $\lambda$ for every component in a hydraulic system. Using thus calculated $\lambda$, the expected reliability is derived for all three sub-systems (accumulation, extrusion, and filling sub-system) that comprise the considered hydraulic system. Using thus calculated reliability for all three sub-systems, it is possible to calculate the value for expected reliability, and mean operational time of the entire hydraulic system. For the serially arranged system components, reliability is calculated according to [6] and [7]:

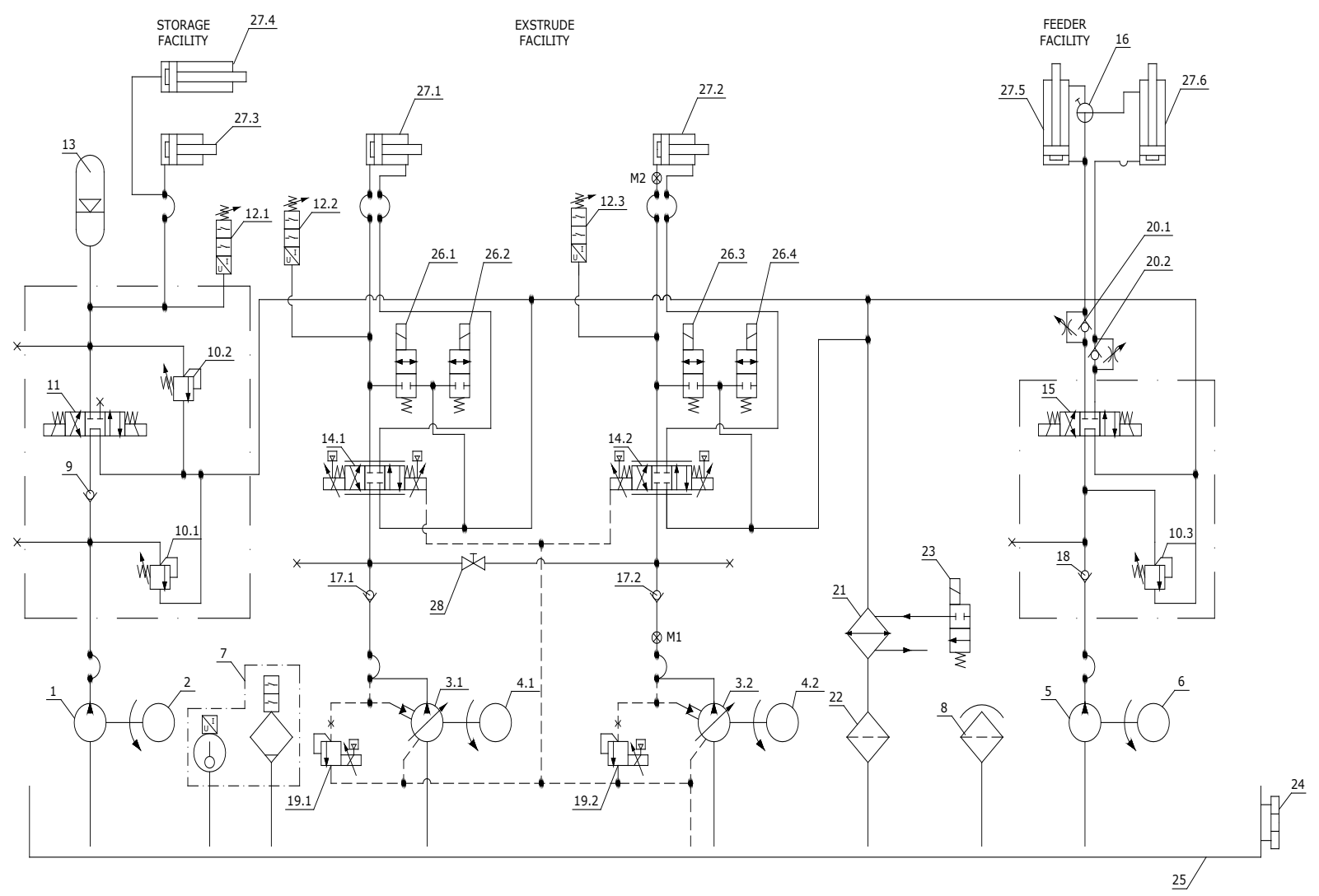

Fig. 1. Block diagram of the hydraulic system used for reliability-analysis-based component design 
Table 1. Reliability of constituent components within the considered hydraulic system [1]

\begin{tabular}{|c|c|c|c|}
\hline \multirow{2}{*}{ Position } & \multirow{2}{*}{ Hydraulic Component (Failure Rate - marks) } & \multicolumn{2}{|c|}{ Failure Rate $\left(\times 10^{-6}\right)$} \\
\hline & & Range of Value & Mean \\
\hline$(1)$ & $(2)$ & $(3)$ & $(4)$ \\
\hline (1), (5) & Gear pump $(\lambda 1, \lambda 5)$ & & 13 \\
\hline (2), (4.1), (4.2), (6) & Electrical motor $(\lambda 2, \lambda 4, \lambda 6)$ & 2 to 18 & 10 \\
\hline$(3.1),(3.2)$ & Axial piston pump $(\lambda 3)$ & 6 to 13 & 9 \\
\hline (9), (17.1), (17.2), (18) & Check valve $(\lambda 9, \lambda 17, \lambda 18)$ & 0.112 to 10.2 & 6.5 \\
\hline$(10.1),(10.2),(10.3)$ & Relief valve $(\lambda 10)$ & 1.41 to 8.13 & 5.88 \\
\hline (11), (15), (23), (26.1), (26.2), (26.3), (26.4) & Electro - hydraulic valve $(\lambda 11, \lambda 15, \lambda 23, \lambda 26)$ & 2.27 to 19.7 & 11 \\
\hline (12.1), (12.2), (12.3) & Pressure switch $(\lambda 12)$ & & 10 \\
\hline 13 & Gas - charged accumulator $(\lambda 13)$ & 0.35 to 7.5 & 6.8 \\
\hline (14.1), (14.2) & Proportional valve $(\lambda 14)$ & 16.8 to 56 & 30 \\
\hline (16), (28) & Ball valve $(\lambda 16, \lambda 28)$ & 1.11 to 7.6 & 4.6 \\
\hline (19.1), (19.2) & Pressure compensated relief valve $(\lambda 19)$ & & 20 \\
\hline (20.1), (20.2) & Check valve - flow restrictor $(\lambda 20)$ & 0.112 to 10.2 & 6.5 \\
\hline 21 & Radiator for cooling $(\lambda 21)$ & & 1 \\
\hline 22 & Filter $(\lambda 22)$ & 0.01 to 1.62 & 0.79 \\
\hline 25 & Hydraulic reservoir $(\lambda 25)$ & 0.48 to 2.52 & 1.5 \\
\hline (27.1), (27.2), (27.3), (27.4), (27.5), (27.6), & Hydraulic cilinders $(\lambda 27)$ & & 0.008 \\
\hline & redesigned hydraulic system & & \\
\hline (29.1), (29.2) & Gear pump with frequency regulation $(\lambda 29)$ & & 13 \\
\hline$(30.1),(30.2)$ & Relief valve - with relieve of load valve $(\lambda 30)$ & 0.224 to 14.1 & 5.7 \\
\hline$(31.1),(31.2)$ & Electro - hydraulic valve (directional control) $(\lambda 31)$ & 1.81 to 7.22 & 4.6 \\
\hline
\end{tabular}

$$
R(t)=R_{1}(t) \cdot R_{2}(t) \ldots R_{n-1}(t) \cdot R_{n}(t)=\prod_{i=1}^{n} R_{i}(t) .
$$
[7]:

Substituting Eq. (1) into Eq. (2), yields [6] and

$$
R(t)=e^{-\lambda_{1} \cdot t} \cdot e^{-\lambda_{2} \cdot t} \cdots e^{-\lambda_{n-1} \cdot t} \cdot e^{-\lambda_{n} \cdot t}=e^{-\left(\sum_{i=0}^{n} \lambda_{i}\right) \cdot t} .
$$

Eq. (3) is used for the calculation of reliability for all three sub-systems in particular, by substituting each failure rate value, $\lambda_{i}$, with the corresponding value from Table 1.

The time interval, $t$, is taken from the range of values: $1,2,3,6,9,12,18,24,30$, and 36 months of operation, that is, $730,1460,2190,4380,6570$, $8760,13140,17520,21900$, and 26280 hours for each particular sub-system.

Mean times of hydraulic system components listed in the fourth column of Table 1 are taken for failure rates $\left(\lambda_{i}\right)$.

The calculated expected reliabilities for each of the sub-systems, for the time interval of $t=730$ hours (1 month) are stated below:

a) Filling sub-system $R(730)=0.957$,

b) Extrusion sub-system $R(730)=0.781$, c) Accumulation sub-system $R(730)=0.976$.

The same is applied to the calculation of other time intervals.

The values above indicate that the system's designer has made a mistake when selecting the system components since one of the sub-systems has got incomparably lower reliability, thus significantly degrading the potential performance of the whole system. In other words, the other two sub-systems feature high quality and costly components which boost system price without significantly improving its performance.

The charts of change of each component's reliability for every time interval are shown in Figs. 2 to 4 .

Total reliability of the hydraulic system has been calculated and charted (Fig. 5) for the considered filling, extruding, and accumulating sub-systems.

Analysis of the charts leads to the conclusion that the sub-system for extrusion represents the least reliable sub-assembly within the hydraulic system (Figs. 3 and 5), i.e. the components with failure rates $\lambda_{3}, \lambda_{14}$, and $\lambda_{19}$, exhibit highest unreliability. 


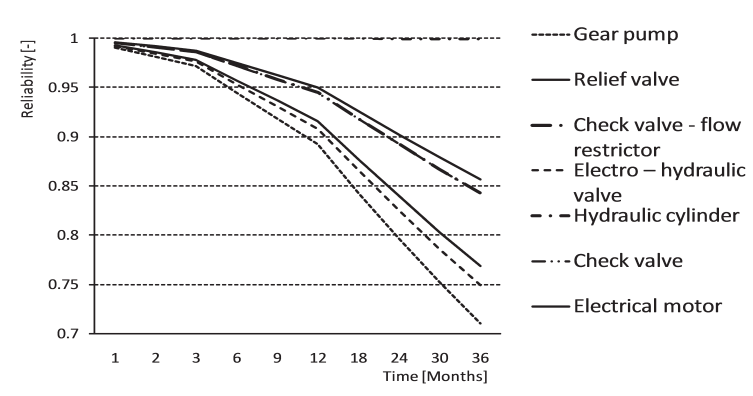

Fig. 2. The change in reliability of hydraulic components of the filling sub-system

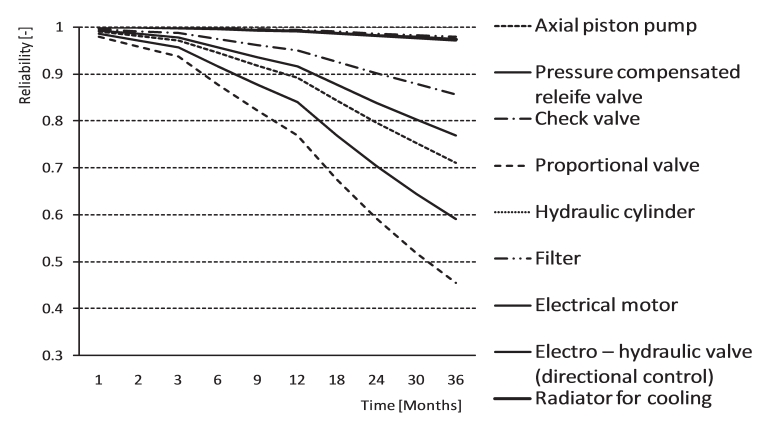

Fig. 3. The change in reliability of hydraulic components of the extruding sub-system

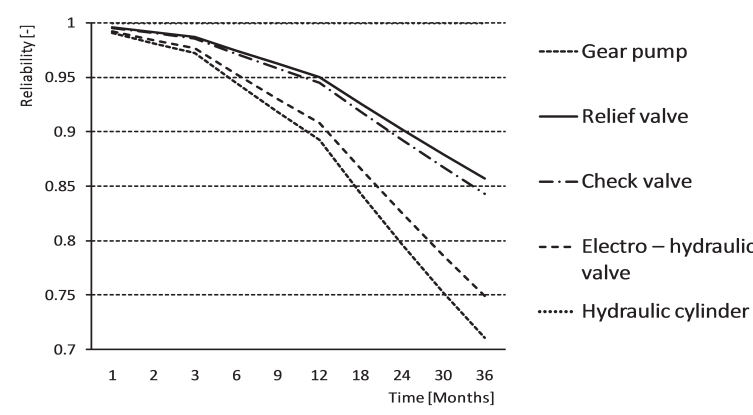

Fig. 4. The change in reliability of hydraulic components of the accumulating sub-system

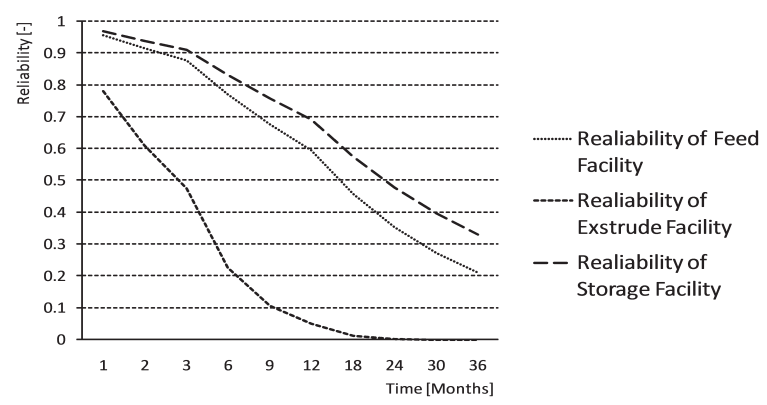

Fig. 5. Total reliability of the three sub-systems of the considered hydraulic system

Based on these results, the logical conclusion would be to redesign the extrusion sub-system in order to increase its reliability, and to replace unreliable components - in this case, the axial piston pump with proportional pressure relief valve, and the proportional distributing valve. The replacement of these components would allow:

- increment of extrusion sub-system's reliability,

- satisfying the system's functional and efficiency requirements.

The expected mean operational time of the system from Fig. 1 is calculated according to following formula, [6] and [7]:

$$
T_{w}=\frac{1}{\lambda}
$$

and, for the total system failure rate of $\lambda=4.41 \times 10^{-4}$, it equals $T_{w}=1 / \lambda=2268.96$.

Thus derived mean operational time represents a theoretical value which indicates that, disregarding the adverse effects of human and process influence, the re-designed hydraulic system should have an average operational time of more than 90 days.

\section{MONITORING THE SYSTEM IN OPERATION}

During the operation of the considered hydraulic system, frequent failures have been observed every two hours of operation. The failures observed do not correspond to the calculated mean operational time ( $T_{w} \approx 2269$ hours), which indicates a serious fault in the hydraulic system. This extreme deviation from the expected mean operational time occurs due to extrusion system overload, i.e. the axial piston pumps, 3.1 and 3.2, suffer from overheating, as well as the electro motors which drive them - position 4. Extruder cylinders, 27.1 and 27.2, are coupled, which means that when the cylinder 27.1 performs its working stroke, the cylinder 27.2 performs its return stroke, and vice versa. Within the two hour interval the entire hydraulic system suffered from failure which lasted for two hours - as was required by the pump and motors to cool down - which reduced the hydraulic system's productivity by $50 \%$. It should be noted that the system is designed to operate 24 hours a day, and breaks are allowed just for the simple replacements of particular components or for preventive actions. Based on the results of reliability analysis of the extruding sub-system components - which indicate that the most critical components in the system are piston axial pump, the proportional pressure relief valve, and the proportional distributing valve - pressure and flow monitoring have been performed in the entire extrusion sub-system, in order to establish the parameters which influence disturbances, as well as the pressure drops in the system [2] to [5]. Monitoring and recording of 
pressure change have been performed at two locations in the hydraulic sub-system of the extruder: after the pump (3.2) at proportional distributing valve (14.2) fluid inlet - measurement spot M1, and after the proportional distributing valve (14.2) at working piston (27.2) fluid inlet - measurement spot M2.

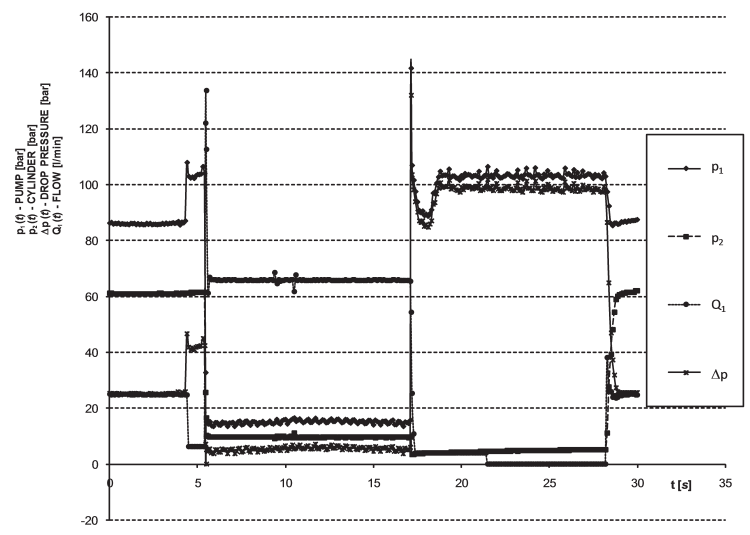

Fig. 6. Change of operating parameters $\left(p_{1}, p_{2}, Q_{1}\right.$ and $\left.\Delta p_{1}\right)$ in the extruder hydraulic system

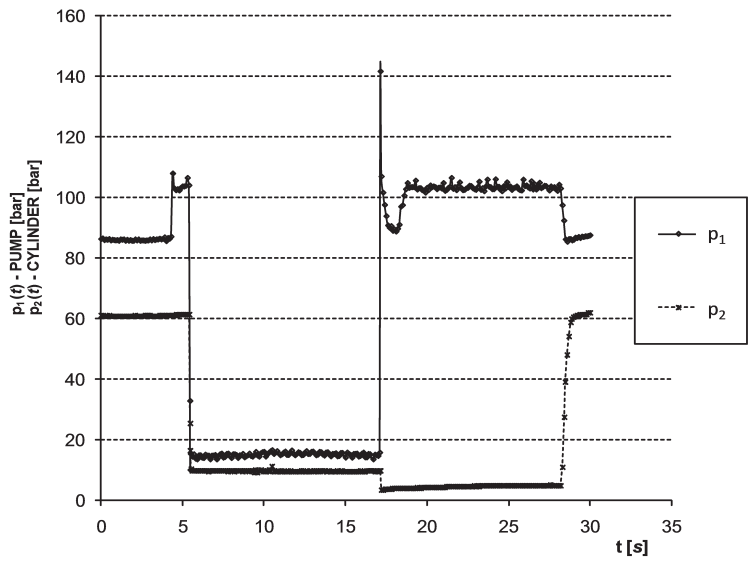

Fig. 7. Change of pressures $p_{1}$ and $p_{2}$ in the extruder hydraulic system within a 20 ms time interval

The recorded working parameters ( $p_{1}$ [bar] pump pressure, $p_{2}[\mathrm{bar}]$ - cylinder pressure, $Q_{1}[1 / \mathrm{min}]$ - flow, and $\Delta p_{1}$ [bar] - pressure drop between pump and cylinder) have been charted and shown in Figs. 6 to 8 .The hydraulic system requires a constant fluid feed with relatively small pressure fluctuations during the operation. However, the monitoring of pressure and flow in the extrusion hydraulic sub-system has revealed substantial oscillations of pressure and flow. These oscillations cause increase in temperature of working fluid, working components (primarily the drive pump), while also causing overheating of the drive electro motors. The pressure peaking to 3421 bar, which exceeds the allowed values for this system and its pump, is shown in Fig. 8. Based on the data partially presented in this work, it can be concluded that the extruder hydraulic sub-system's design is flawed.

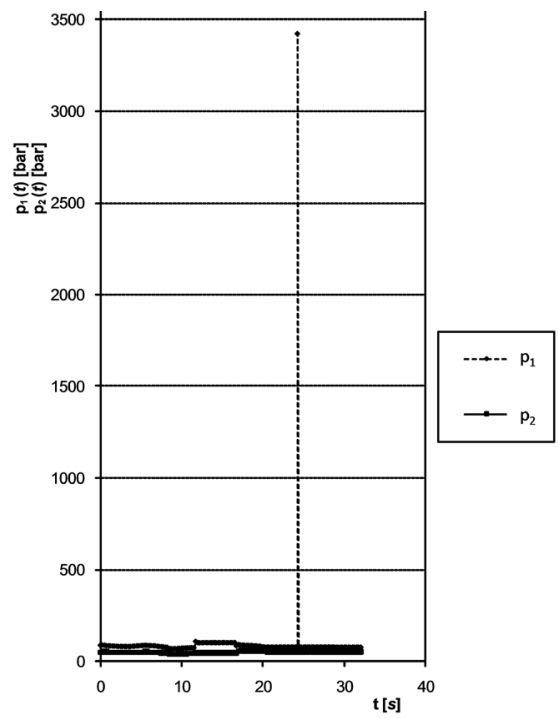

Fig. 8. Pressure $p_{1}$ peaks to 3421 bar within the 10 ms interval

That is, having the primary system requirement in mind, i.e. the necessity for constant flow distribution from the pump to the actuators, and constant operating pressure in all actuators (cylinders 27.1 and 27.2), the design concept has been completely wrong.

Comparing the reliability analyses for the system in hand, as well as the monitoring performed on the system in full operation, the following can be concluded: the axial piston pump in the system provides the required capacity in the extruder hydraulic system, but does not require the capacity regulation by the proportional pressure valve, as shown in Fig. 1.

In addition, the proportional distributing valve has been used in an entirely wrong way, bearing in mind that the extruder sub-system requires maximum fluid quantity to supply the actuator. This leads to a conclusion that instead of proportional distributing valve, a classical electric distribution valve should be used in either fully closed or fully open working position.

An analysis of reliability has shown that the proportional valve is the weakest component not only in the extruder hydraulic sub-system, but also in the entire hydraulic system. The re-design of the original hydraulic system, as well as the replacement of unreliable components by more reliable ones, leads to a hydraulic system of higher reliability and efficiency rate (Fig. 9). 


\section{DESIGN OF A NEW SYSTEM}

The results obtained through the monitoring of the hydraulic system's operating parameters have been used in the design of the improved system (Fig. 9), as well as the results of analysis of expected reliability for the considered hydraulic system. The result has revealed the following critical components in the subsystem for extrusion: the axial piston pump (position 3 ), the proportional pressure relief valve (position 19), and the proportional distributing valve (position 14). These components have been replaced. The axial piston pump (positions 3.1 and 3.2) has been replaced by a gear pump with frequency regulation (positions 29.1 and 29.2); the proportional pressure relief valve (positions 19.1 and 19.2), has been replaced by an internal pressure relief valve (positions 30.1 and 30.2); finally, the proportional distributing valve (positions 14.1 and 14.2) has been replaced by a classical electrohydraulic distribution valve (positions 31.1 and 31.2), Fig. 9. The failure rate values have also been taken from Table 1 for the analysis of expected reliabilities of the considered elements. The analysis of reliability for the newly designed hydraulic system has been performed with an additional assumption that the random variable (operational time) varies according to exponential distribution law (Eq. (1)).

Further analysis of expected reliability of the improved hydraulic system has been performed in the same fashion as the original hydraulic system, using Eqs. (2) and (3). Expected reliability of all components of the three sub-systems has been calculated for the improved system (Fig. 9).

Example: for the $t=730$ hours ( 1 month) time interval, the reliability for all three sub-systems equals:

a) Filling sub-system $R(730)=0.957$,

b) Extrusion sub-system $R(730)=0.955$,

c) Accumulation sub-system $R(730)=0.976$.

Calculation for all other time intervals is performed in the same manner. The charts showing a change in the reliability of each component of the hydraulic system are presented in Figs. 10 to 12, for all time intervals provided by the analysis.

Reliability of the entire hydraulic system is charted in Fig. 13, where all three sub-systems

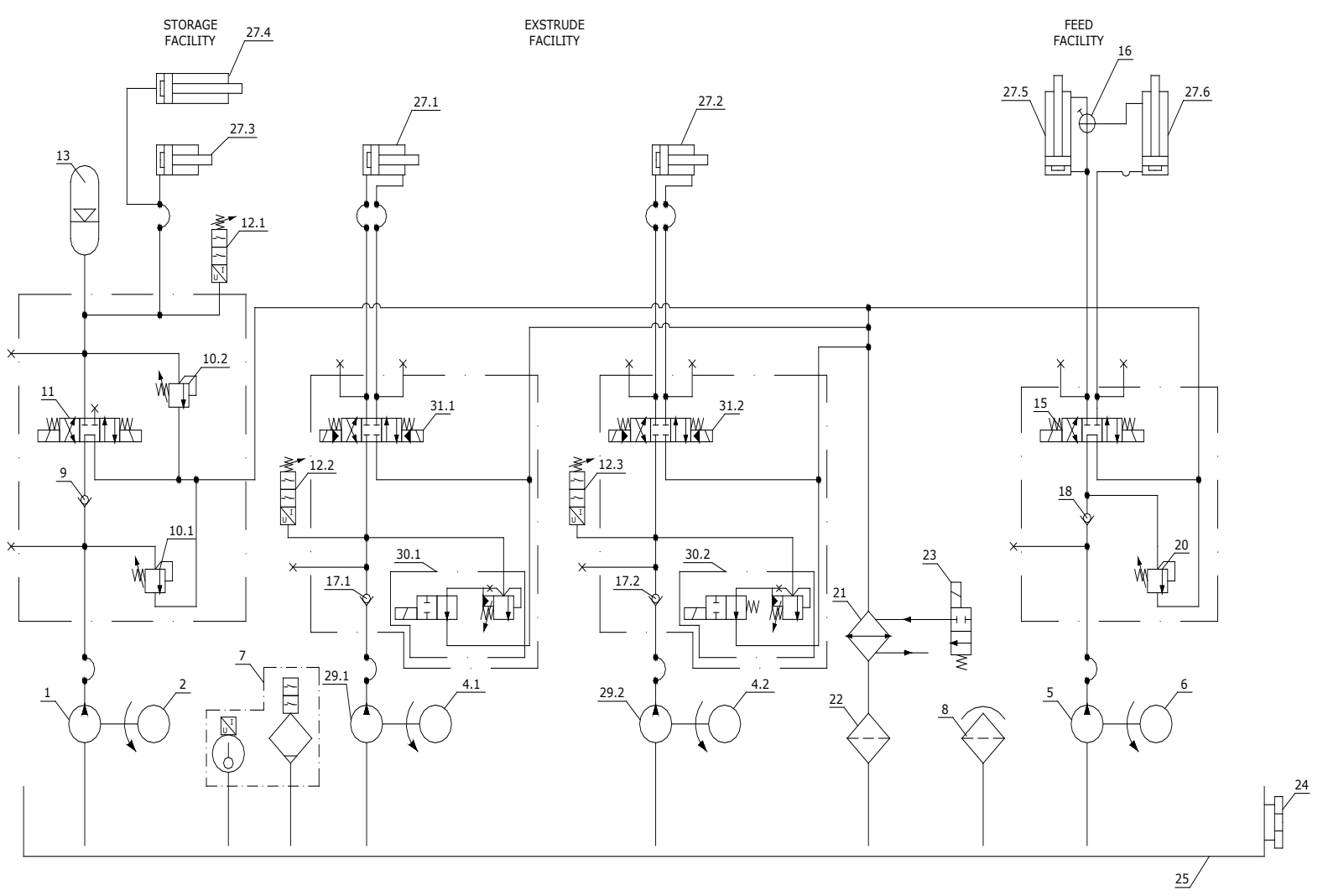

Fig. 9. Block diagram of the improved hydraulic system 
(filling, extruding, and accumulation) define the total reliability of the hydraulic system from Fig. 9.

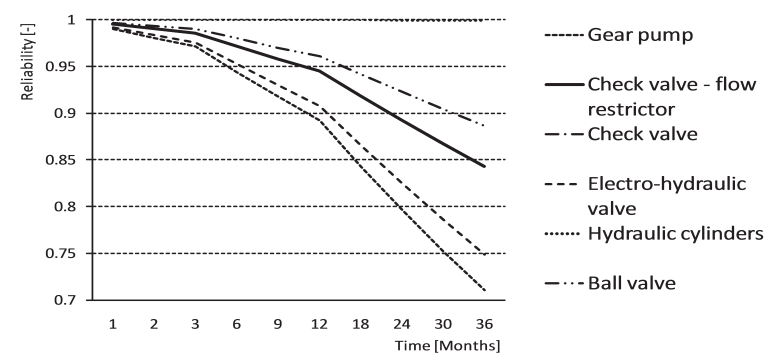

Fig. 10. The change in reliability of hydraulic components of the re-designed filling sub-system

From the standpoint of reliability engineering, the analysis of improved hydraulic system leads to the conclusion that the extruder remains the most unreliable component, i.e. the least reliable assembly of the hydraulic system (Fig. 13).

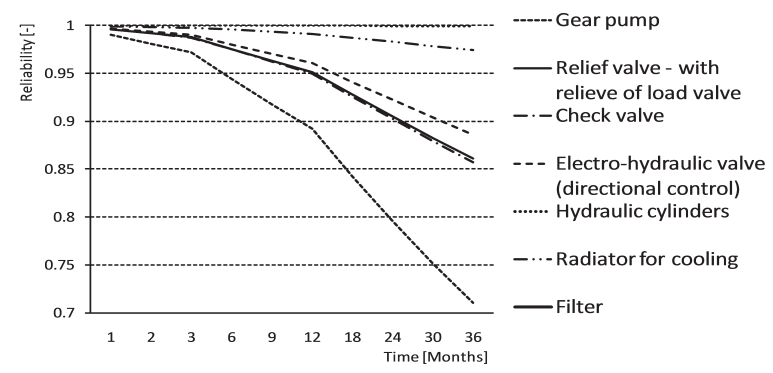

Fig. 11. The change in reliability of hydraulic components of the re-designed extruding sub-system

However, compared to the original system, the reliability gain of $17.43 \%$ represents a significant improvement.

The replacement of axial piston pump by a gear pump, as well as the replacement of proportional distributing valve with a classical electro-hydraulic valve, has lead to the increase of total reliability of the entire hydraulic system compared to the original solution.

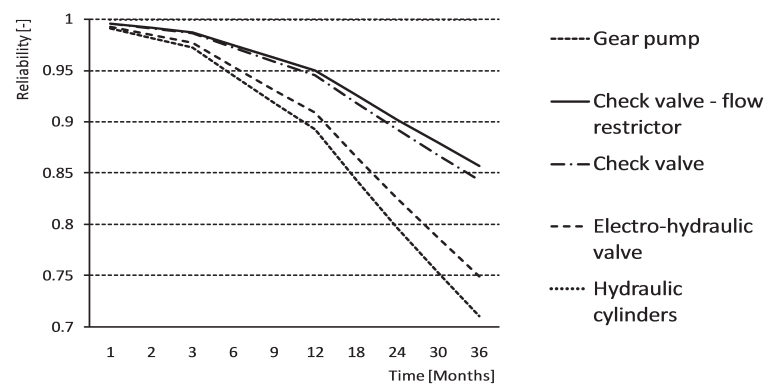

Fig. 12. The change in reliability of hydraulic components of the re-designed accumulating sub-system
The mean operational time for the improved system equals $T_{w}=1 / \lambda=5811.99 \mathrm{~h}$ which indicates an increase of $156 \%$ in comparison with the original system. After redesigning, the hydraulic system was again put into a 24-hour operating regime.

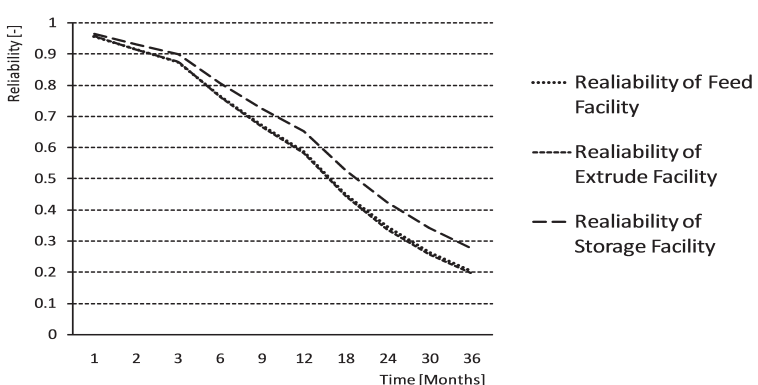

Fig. 13. Reliability of all three assemblies of the re-designed hydraulic system

During exploitation, failures due to overheating were completely eliminated (compared to previous situation when they occurred in 2 hour intervals).

Other types of failures have not been observed to this moment. This can be attributed to improved reliability, as well as to the preventive system maintenance procedure, which has significantly reduced the negative influence of humans and working conditions. The only observed down times in the system are due to machine setups to accommodate a change of production type.

\section{CONCLUSION}

Analysis of results obtained by a combined application of the reliability theory and monitoring of operating parameters of the considered hydraulic system has lead us to the following conclusions:

- The reliability theory helps identifying the weakest - most critical components in a hydraulic system (which can also be concluded by monitoring the operation of a hydraulic system and analyzing failure data). However, system monitoring alone allows neither determination of deviation between the expected and real reliability of all system components, nor the influence of lower reliability on the reliability of the entire hydraulic system. Reliability analysis estimates reliability of system components, and their ranking and charting, as shown in Figs. 2 to 4 and 10 to 12 ,

- monitoring of operating parameters enables the identification of down-time causes and failures of particular system components (monitoring of operating system parameters, such as the 
pressure, flow, working fluid temperature, key components temperature, etc. reveals the most critically loaded system components, as well as the values of operating parameters, which can lead to component down-times or failures),

- adequate replacement of critical elements in a hydraulic system, accompanied by a proper selection of components, not only increases the total system reliability, but also prolongs the mean operational time (i.e., system productivity and efficiency),

- continual monitoring of operating parameters in a hydraulic system, i.e. automated control of system operating parameters, makes it possible to predict and influence system reliability.

The general conclusion that can be drawn is that reliability analysis is useful in conjunction with the monitoring of operating parameters of hydraulic system and its components during exploitation, as well as during the designing of a novel hydraulic system. Unfortunately, designing hydraulic systems based on reliability analysis is still not widespread enough. This can be attributed to the fact that in many cases the designers lack sufficient knowledge of the features and advantages of reliability analysis in the design process. The monitoring of operating parameters of a hydraulic system in conjunction with reliability analysis allows the critical system components to be singled out and improved in terms of reliability and efficiency, once the hydraulic system is designed and put into operation.

With this in mind, this paper should draw the hydraulic systems designers' attention towards reliability analysis, which has the potential to increase efficiency of hydraulic systems.

\section{NOMENCLATURE}

$p \quad$ pressure [bar]

$\Delta p$ pressure drop [bar]

$Q \quad$ flow [1/min]

$R \quad$ system reliability [-]

$t$ operational time [h]

$T_{W}$ mean operational time [h]

$\lambda$ failure rate $\left[\mathrm{h}^{-1}\right]$

\section{REFERENCES}

[1] Fitch, E.C., Hong, I.T. (2004). Hydraulic System Design for Service Assurance. BarDyne, Inc., Stillwater.

[2] Savić, V., Knežević, D., Lovrec, D., Jocanović, M., Karanović, V. (2009). Determination of pressure losses in hydraulic pipeline systems by considering temperature and pressure. Strojniški vestnik - Journal of Mechanical Engineering, vol. 55 , no. 4, p. 237-243.

[3] Savić, V., Zirojević, Lj. (2003). Oil hydraulics 3. IKOS, Novi Sad. (in Serbian)

[4] Jocanović, M. (2010). Approach to research and define the model for the calculation of flow of solid particles with a mass of mineral oil through the gaps in a function of the constructive operating parameters of hydraulic components, $\mathrm{PhD}$ dissertation, University of Novi Sad, Novi Sad. (in Serbian)

[5] Herakovič, N. (2009). Flow - force analysis in a hydraulic sliding-spool valve. Strojarstvo, vol. 51, no. 6, p. 555-564.

[6] Birolini, A. (2010). Reliablity Engineering Theory and Practice, $6^{\text {th }}$ ed.. Springer, Berlin.

[7] Ivanović, G., Stanivuković, D., Beker, I. (2010). Reliability of technical systems. Faculty of Technical Sciences Publishing, Novi Sad. (in Serbian) 